\title{
Design of Multi-channel Wavelength Demultiplexer Based on Asymmetrically Coupled Nanodisk Resonators
}

\author{
Weici Liu ${ }^{1,2}$, Faqiang Wang ${ }^{3 *}$ and Ruisheng Liang ${ }^{3}$ \\ ${ }^{1}$ Department of Electronic Information Engineering, Guangzhou College of Technology and Business, Foshan 528138, China \\ ${ }^{2}$ School of physics and Telecommunication Engineering, South China Normal University, Guangzhou 510006, China \\ ${ }^{3}$ Laboratory of Nanophotonic Functional Materials and Devices (SIPSE), and Laboratory of Quantum Engineering and Quantum \\ Materials, South China Normal University, Guangzhou 510006, China \\ ${ }^{*}$ Corresponding author
}

\begin{abstract}
A novel multi-channel wavelength demultiplexer (WDM) consisting of metal-insulator-metal waveguides and asymmetrically coupled nanodisk resonators is designed and simulated. The results show the WDM can export any single mode while undesirable modes are suppressed.
\end{abstract}

Keywords-WDM; waveguides; resonators; FDTD

\section{INTRODUCTION}

Surface Plasmon polaritons (SPPs) are attractive for the good performance in overcoming the traditional diffraction limit and light-manipulation in sub-wavelengthscales[1]. Several metal-insulator-metal (MIM) waveguides based on SPPs have been proposed recently[2], such as filters, splitters, sensors, Bragg-reflectors, Mach-Zehnder interferometers, all-optical switches, Y-shaped combiners and modulators. Filters are extraordinarily important branch and various structures have been investigated. The wavelength selective devices are crucial component in the optical computing and telecommunication as their symmetry, simplicity, and ease of fabrication. A new type of MIM structure to realize multi-channel wavelength-selective function in WDM system appeared recently based on previous filters structure, such as tunable multi-channel wavelength demultiplexer based on disk-shaped nanocavities[3,4]. Compared with the multi-channel plasmonic filter with the MIM Fibonacci-sequence gratings [5], these filters are more appropriate for high integration in nanometer scales due to their simple and compact structure.

In this paper, we propose a new kind of multi-channel wavelength demultiplexer based on MIM waveguides andasymmetrically coupled nanodisk resonators, and simulate the transmission properties by finite-difference time-domain (FDTD). Based on this structure, four ports can separate four resonant modes simultaneously, which can be adjusted by changing the structure parameters.

\section{THEORY AND MODEL}

$$
\varepsilon_{m}(m)=\varepsilon_{\infty}-\frac{\omega_{0}^{2}}{\omega(\omega+i \gamma)}
$$

Where $\varepsilon_{\infty}=3.7$ is the dielectric constant at infinite angular frequency, ${ }^{\omega_{0}}$ means the bulk plasma frequency with value of 9.1 eV, which stands for the natural frequency of the oscillations of free conduction electrons, $\gamma$ represents the damping frequency of the oscillations with value of $0.018 \mathrm{eV}$. $\omega$ is the angular frequency of the incident light.

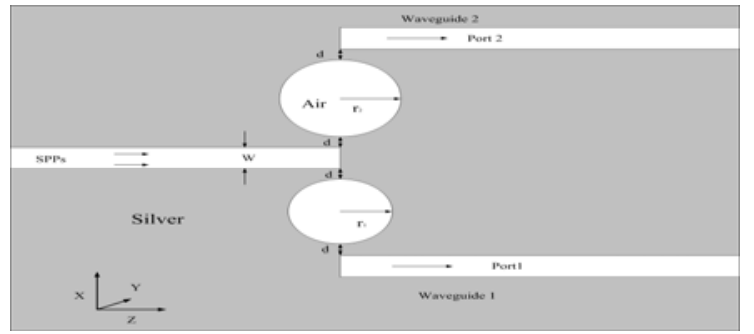

FIGURE I. THE SCHEMATIC DIAGRAM OF THE STRUCTURE

$$
\text { WITH } r_{1}, r_{2}=340,410 \mathrm{~nm} ; \mathrm{w}=50 \mathrm{~nm} ; \mathrm{d}=10 \mathrm{~nm}
$$

Figure I indicates the schematic diagram of the demultiplexer that waveguides and nanodisk resonators are asymmetrically coupled with each other. The width of waveguides $\mathrm{w}$ is set to be $50 \mathrm{~nm}$, and the radius of nanodisk resonators are set to be $340 \mathrm{~nm}$ and $410 \mathrm{~nm}$ respectively. The gaps between the waveguides and the nanodisk resonators are set to be $10 \mathrm{~nm}$. The dielectric in the waveguides is air with refractive index equaling 1 . And the metal is sliver, whose frequency-dependent complex relative permittivity is described by the Drude model [6]. When the incident optical wave transmits through input waveguide, it is partly coupled into the two nanodisk resonators, and then transmits out along the port 1 and port 2. The stable standing wave in the nanodisks will be formed when resonance condition is reached, which can be described by following equation:

$$
k_{d} \frac{H_{n}^{(1)^{\prime}}\left(k_{m} r\right)}{H_{n}^{(1)}\left(k_{m} r\right)}=k_{m} \frac{J_{n}^{\prime}\left(k_{d} r\right)}{J_{n}\left(k_{d} r\right)}
$$

where $k_{d m}=k\left(\varepsilon_{d m}\right)^{1 / 2}$ is the wave vectors in the dielectric nanodisks and metal, and $r$ is the radius of the nanocavity. 
$\varepsilon_{m}$ is the relative permittivity of the metal. $\varepsilon_{d}$ is the relative permittivity of the dielectric. $k$ is the wave number and contains a relatively small negative imaginary part which represents the loss. ${ }^{J_{n}}$ and ${ }^{J_{n}^{\prime}}$ are Bessel function with the order n and its derivation, $H_{n}^{(1)}$ and $H_{n}^{(1)^{\prime}}$ are Hankel function with the order $\mathrm{n}$ and its derivation. Here, the first and second order modes in the nanodisks correspond to the first and second order of Bessel and Hankel functions, respectively. From Eq. (2), it shows that the resonance wavelength $\lambda_{0}$ is confined by radius $r$ and the refractive index.

According to the coupled mode theory, the transmission of the band-pass demultiplexer can be written as

$$
T(w)=\frac{\left(1 / \tau_{w}\right)^{2}}{\left(w-w_{0}\right)^{2}+\left(1 / \tau_{i}+1 / \tau_{w}\right)^{2}}
$$

Where $W$ is the frequency of the incident light. $w_{0}$ is the resonance frequency. $\tau_{w}$ is the decay rate of the field refers to the energy loss in the cavity through the waveguide and $\tau_{i}$ means the decay rate coming from internal loss in the resonator. From Eq. (3), it can be seen that, when the incident light $w$ is far from resonance frequency $w_{0}, T$ nearly equals to zero and that is to say, the incident light will be reflected completely. Besides, the resonance peak transmittance $T_{M A X}=\left(1 / \tau_{w}\right)^{2} /\left(1 / \tau_{i}+1 / \tau_{w}\right)^{2}$ is close to unity when $\tau_{i}$ is far less than $\tau_{w}$.

\section{SiMULATION AND RESULTS}

The properties of the SPP propagation are simulated by two-dimensional FDTD method. And the absorbing boundary conditions are convolutional perfectly matched layers (CPML). The grid sizes are $\Delta x=\Delta z=5 n m$ and the time step can be described as $c \Delta \leq 1 / \sqrt{(\Delta \mathrm{x})^{-2}+(\Delta z)^{-2}}$.

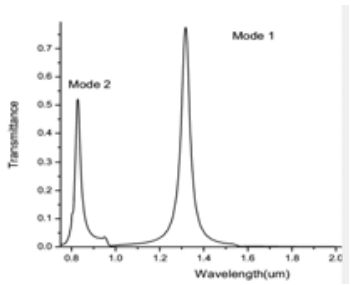

(A)

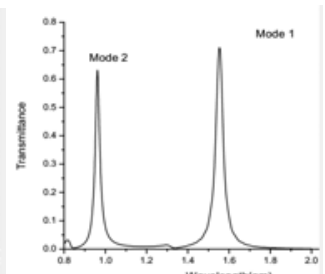

(B)

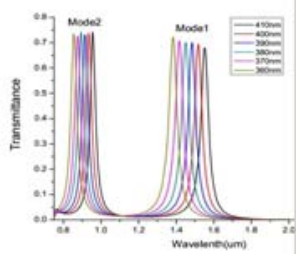

(C)

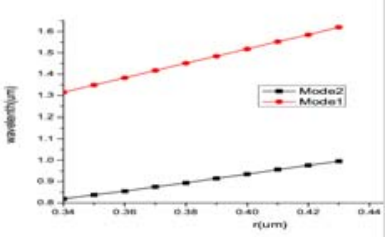

(D)
FIGURE II. (A) THE TRANSMISSION SPECTRUM OF THE PORT 1, (B) THE TRANSMISSION SPECTRUM OF THE PORT 2, (C) THE TRANSMISSION SPECTRUM OF THE PORT 2 FOR DIFFERENT RADII, (D) THE RELATION BETWEEN RESONANCE WAVELENGTHS AND THE RADIUS OF NANODISK

Figure II. (A) and Figure II. (B) illustrate the transmission spectrum of port 1and 2. It is found that there are two resonance peaks at the wavelengths of $850 \mathrm{~nm}$ and $1310 \mathrm{~nm}$ for port 1 , and $980 \mathrm{~nm}$ and $1550 \mathrm{~nm}$ for port 2, respectively. The maximum transmittances corresponding to above modes are $52 \%, 78 \%, 63 \%$ and $71 \%$ respectively. Figure II. (C) illustrates the transmission spectrum of the structure for different radii. Figure II. (D) illustrates that the resonance wavelength has a linear relationship with the radius of nanodisks. And the resonance wavelengths of transmission spectrum exhibit a red-shift with the increasing of radius $r$.

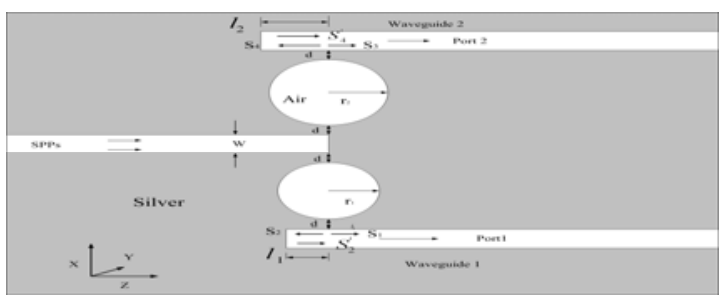

(A)

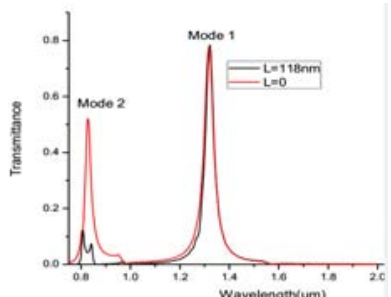

(B)

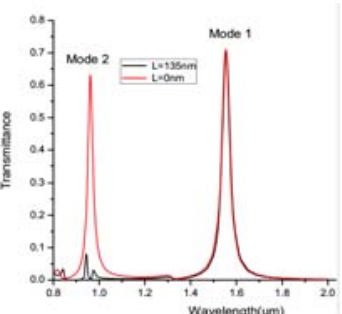

(C)
FIGURE III. (A) THE SCHEMATIC DIAGRAM OF THE ASYMMETIC

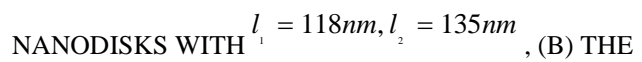
TRANSMITTANCE SPECTRUM OF PORT 1 FOR DIFFERENT EXTRA LENGTH, (C) THE TRANSMITTANCE SPECTRUM OF PORT 2 FOR DIFFERENT EXTRA LENGTH 
We assume the difference of the phase of per round trip in the extra parts is $\Delta \varphi_{1,2}=k_{1,2}(\omega) \times 2 l_{1,2}+\varphi_{11,12}$, where $k_{1,2}(\omega)$ is the angular wave-number of the wavelengths ins the waveguide 1 or $2 . \varphi_{l 1, l 2}$ is phase compensation at the reflected port of the two output waveguides. When satisfied condition $\Delta \varphi_{1,2}=(2 m-1) \pi$, the waves will disappear. For the relation $\operatorname{Re}\left[k_{1,2}(\omega)\right]=2 \pi n / \lambda$, the disappeared waves satisfy relation $\lambda_{1,2}=4 n l_{1,2} /\left[(2 m-1)-\varphi_{11,12} / \pi\right]$, here $n=1.5$ for waveguide $w=50 \mathrm{~nm}$, and assuming phase compensation is $0.15 \pi$. Substituting them and the data $m=1$, $l_{1}=118 \mathrm{~nm}, l_{2}=135 \mathrm{~nm}$ into the formula of $\lambda_{1,2}$, then the wavelengths $850 \mathrm{~nm}$ and $980 \mathrm{~nm}$ disappear.

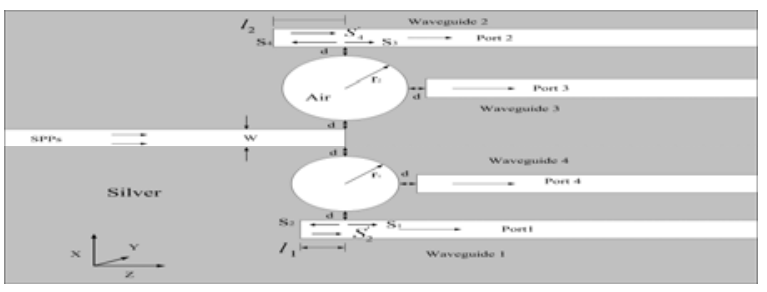

FIGURE IV. THE SCHEMATIC DIAGRAM OF THE $1 \times 4$ WDM

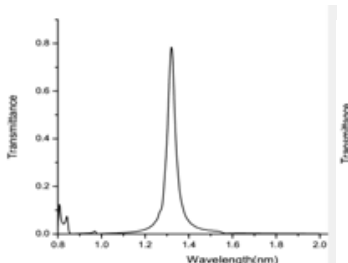

(A)

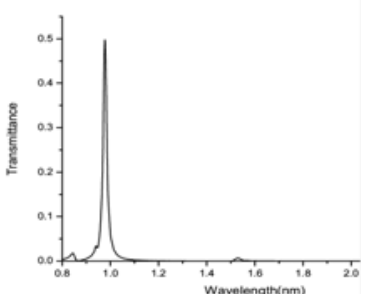

(C)

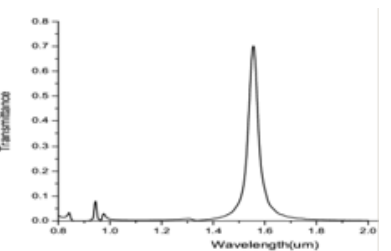

(B)

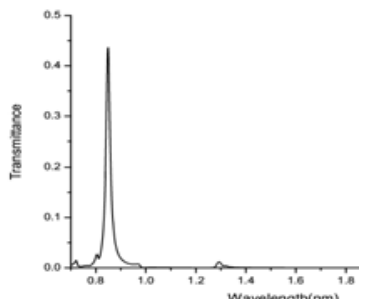

(D)
FIGURE V. THE TRANSMITTANCE SPECTRUM OF EACH OUTPUT PORT, (A)CORRESPOND TO THE PORT 1 AND RESONANCE WAVELENGTH $\lambda_{0}=1310 \mathrm{~nm}$,(B) CORRESPOND TO THE PORT 2 AND $\lambda_{0}=1550 \mathrm{~nm}$,(C) CORRESPOND TO THE PORT 3 AND $\lambda_{0}=980 \mathrm{~nm}$,(D) CORRESPOND TO THE PORT 4 AND $\lambda_{0}=850 \mathrm{~nm}$

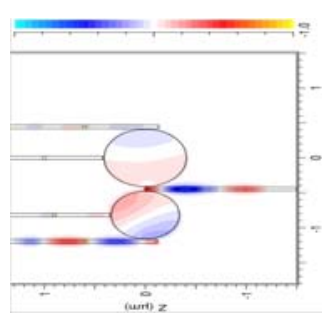

(A)

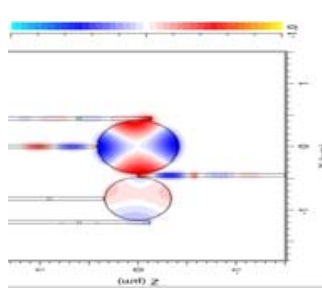

(C)

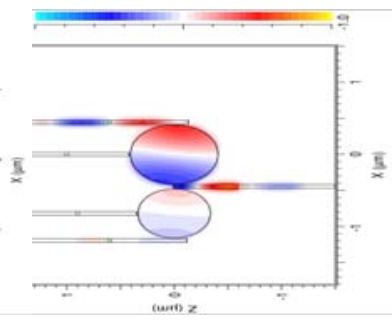

(B)

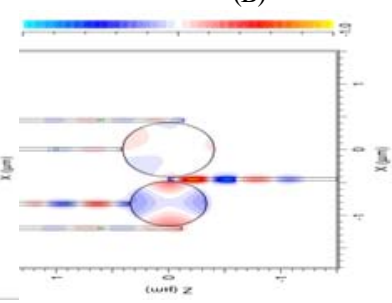

(D)
FIGURE VI. THE MAGNETIC FIELDS INSIDE EACH PO (A) THE PORT $1 \mathrm{AND} \lambda_{0}=1310 \mathrm{~nm}$, (B) THE PORT 2 AND $\lambda_{0}=1550 \mathrm{~nm}$, (C) THE PORT 3 AND $\lambda_{0}=980 \mathrm{~nm}$, (D) THE PORT 4 AND $\lambda_{0}=850 \mathrm{~nm}$

In figure $\mathrm{IV}$, based on above results, a structure of multi-channel WDM is designed. It comprised of one input waveguide and four output waveguides. Where two ports respectively at the middle of two nanodisks (waveguide 3 and 4 ), and the other two with inhibiting properties are at the bottom of nanodisk 1 (waveguide 1) and the top of nanodisk 2 (waveguide 2). One can see from figure $\mathrm{V}$, that the four modes inside the two nanodisks are separated, and the resonance modes around $1310 \mathrm{~nm}, 1550 \mathrm{~nm}, 980 \mathrm{~nm}$, and $850 \mathrm{~nm}$ are coupled to different output waveguides, ports 1- 4, respectively. In figure VI, we give the propagation of the field $\left|H_{z}\right|$ for port 1-4. The coupling loss between the nanodisk resonators and MIM waveguide is about $2.57 \mathrm{~dB}$. The propagation loss of MIM waveguide is about $0.41 \mathrm{~dB} / \mu \mathrm{m}$. The crosstalk are $-13.76 \mathrm{~dB},-13.84 \mathrm{~dB},-14.61 \mathrm{~dB}$ and $-14.98 \mathrm{~dB}$ for the channels of $850 \mathrm{~nm}, 980 \mathrm{~nm}, 1310 \mathrm{~nm}$ and $1550 \mathrm{~nm}$, respectively.

\section{CONCLUSION}

In conclusion, a novel multi-channel wavelength demultiplexer consisting of MIM waveguides and several nanodisk resonators asymmetrically coupled is proposed and numerically simulation by FDTD method. By adjusting the radius of the nanodisk, the desirable resonance wavelengths can be easily gotten. The resonance modes inside nanodisk can be easily inhibited and separated by adding a segment of waveguide at the input/output waveguide. Our results are better about 30\% than that of the structure in Ref [2]. The mechanism of our scheme is based on the near-field coupling between nano-disk resonators and MIM waveguide, which is different from that of the scheme [7] based on the phase coupling and multiple electromagnetically induced transparencies (EIT)-like spectral responses in graphene metamaterials consisting of a series of self-assembled graphene Fabry-Pérot (FP) cavities. And the central wavelength of multi-channel-selective filters in Ref. [7] is within mid-infrared, while the central wavelength of our scheme is within optical communication band in 
near-infrared. The designed WDM can export single mode in four waveguides, and the output of undesirable modes can be suppressed, and this is the first time to get the four single mode by the two nanodisk resonators. In addition, if needed, we can add much more resonators to get more than four single modes though the transmission will be reduced repectively. Our structure plays an important role in highly integrated optical circuits.

\section{ACKNOWLEDGEMENTS}

This work was supported by the National Natural Science Foundation of China(Grants No. 61275059 and No.61774062); Foundation for Distinguished Young Talents in Higher Education of Guangdong, China, Grants No. 2016KQNCX195), and 2016 Natural Science Project of Guangzhou College of Technology and Business (Grants No. KA201625).

\section{REFERENCES}

[1] W. L. Barnes, A. Dereux, and T. W. Ebbesen, "Surface plasmon subwavelength optics," Nature (London) 424, 824-830 (2003).

[2] J. Tao, X. G. Huang, and J. H. Zhu, “A wavelength demultiplexing structure based on metal-dielectric-metal plasmonic nano-capillary resonators,” Opt. Express 18(11), 11111-11116 (2010).

[3] Wang, H. Lu, X. Liu, D. Mao, and L. Duan, "Tunable multi-channel wavelength demultiplexer based on MIM plasmonic nanodisk resonator at telecommunication regime," Opt. Express 19, 3513-3518 (2011)

[4] Yongkang Gong;Xueming Liu;Leiran Wang .High-channel-count plasmonic filter with the metal-insulator-metal Fibonacci-sequence gratings[J].Optics Letters,2010(3):285-287

[5] Z. Yu, R. Liang, P. Chen, Q. Huang, T. Huang, and X. Xu, "Integrated Tunable Optofluidics Optical Filter Based on MIM Side-Coupled-Cavity”,Plasmonics 7(4), 603-607 (2012).

[6] Q. Li, T. Wang, Y. K. Su, M. Yan, and M. Qiu, "Coupled mode theory analysis of mode-splitting in coupled cavity system,” Opt. Express 18(8), 8367-8382 (2010).

[7] C. Zeng, Y. D. Cui, and X. M. Liu,” Tunable multiple phase-coupled plasmon-induced transparencies in graphene metamaterials". Opt. Express 23(1), 545-551 (2015). 